\title{
In vitro Activity of Tigecycline, a New Glycylcycline, Tested Against 1,326 Clinical Bacterial Strains Isolated from Latin America
}

Ana C. Gales ${ }^{*}$, Ronald N. Jones ${ }^{2}$, Soraya S. Andrade ${ }^{1}$, Andrea S. Pereira ${ }^{1}$ andHélio S. Sader ${ }^{2}$

\author{
Federal University of São Paulo, Division of \\ Infectious Diseases ${ }^{1}$, Department of Medicine, São \\ Paul1, Brazil; The JMI Laboratories², North Liberty, \\ IA, USA.
}

\begin{abstract}
The in vitro activity of tigecycline (former GAR-936), a new semisynthetic tetracycline, was evaluated in comparison with tetracycline and other antimicrobial agents. Material and Methods: A total of 1,326 contemporary clinical isolates collected from the Latin American region were collected in 2000-2002 period and tested with microdilution broth according to the CLSI guidelines. The bacterial pathogens evaluated included Staphylococcus aureus (505), Streptococcus pneumoniae (269), coagulase-negative staphylococci (CoNS; 227), Haemophilus influenzae (129), Enterococcus spp. (80), Moraxella catarrhalis (54), $\beta$-haemolytic streptococci (28), viridans group streptococci (26), and Neisseria meningitidis (8) Results: Tigecycline demonstrated excellent activity against all Gram-positive cocci, with $90 \%$ of penicillin-resistant $S$. pneumoniae strains being inhibited at $0.12 \mu \mathrm{g} / \mathrm{mL}$, while the same isolates had an $\mathrm{MIC}_{90}$ of $>16 \mu \mathrm{g} / \mathrm{mL}$ for tetracycline. All Enterococcus spp. were inhibited at $0.25 \mu \mathrm{g} / \mathrm{mL}$ of tigecycline. Tigecycline $\left(\mathrm{MIC}_{50}, 0.25 \mu \mathrm{g} / \mathrm{mL}\right.$ ) was eight-fold more potent than minocycline $\left(\mathrm{MIC}_{50}, 2 \mu \mathrm{g} / \mathrm{mL}\right.$ ) against oxacillin-resistant $S$. aureus (ORSA); all ORSA were inhibited at $\leq 2 \mu \mathrm{g} / \mathrm{mL}$ of tigecycline. Tigecycline demonstrated excellent activity $\left(\mathrm{MIC}_{50}, 0.5 \mu \mathrm{g} / \mathrm{mL}\right)$ against CoNS with reduced susceptibility to teicoplanin (MIC, $16 \mu \mathrm{g} / \mathrm{mL}$ ). Tigecycline also showed high potency against respiratory pathogens such as $M$. catarrhalis $\left(\mathrm{MIC}_{50}, 0.12 \mu \mathrm{g} / \mathrm{mL}\right)$ and $\mathrm{H}$. influenzae $\left(\mathrm{MIC}_{50}, 0.5\right.$ $\mu \mathrm{g} / \mathrm{mL}$ ). No tigecycline resistant isolates were detected when the proposed susceptible breakpoints $(\leq 4 \mu \mathrm{g} / \mathrm{mL})$ was applied. Conclusions: This results indicate that tigecycline has potent in vitro activity against clinically important pathogenic bacteria, including Gram-positive isolates resistant to both tetracycline and minocycline.

Key Words: Antimicrobial susceptibility, tigecycline, Latin America and SENTRY.
\end{abstract}

The tetracyclines have been widely used during in last four decades, due to their broad-spectrum of antimicrobial activity against Gram-positive and Gram-

Received on 17 May 2005; revised 27 September 2005.

Address for correspondence: Dr. Ana Cristina Gales, MD, PhD. Laboratório Especial de Microbiologia Clínica, Division of Infectious Diseases, Universidade Federal de São Paulo. Rua Leandro Dupret, 188, Zip code: 04025-010 São Paulo - SP Brazil. Email:galesac@aol.com.Fax:+55(11)55715180. Phone: + 55 (11) 5081 2819. This study was presented in part at the 43rd Interscience Conference on Antimicrobial Agents and Chemotherapy, Chicago, Illinois. September 14-17, 2003.

The Brazilian Journal of Infectious Diseases 2005;9(5):348-356 (C) 2005 by The Brazilian Journal of Infectious Diseases and Contexto Publishing. All rights reserved. negative aerobic bacteria, including many intracellular pathogens and anaerobic organisms [1,2]. However, indications for the use of tetracyclines have been limited to specific clinical indications, due to the emergence of resistant strains in frequently isolated species, such as Staphylococcus aureus, Enterococcus spp., Streptococcus pneumoniae, and Neisseria gonorrhoeae [1-4].

Tigecycline, former GAR-936, is the first representative of a new class of antimicrobial agents known as glycylcyclines. This compound is a semisynthetic 9-t-butylglycylamido derivative of the minocycline molecule [5]. Although tigecycline acts on the bacterial ribosome by binding sites similar to those of tetracycline, the radical added to position 9 of the 
tigecycline molecule has provided additional steric hindrance features that result in a greater spectrum of activity [6]. Thus, tigecycline has documented activity against tetracycline-resistant (tet-R) Gram-positive and Gram-negative pathogens, refractory to both efflux and ribosomal protection mechanisms [7].

Our main objective was to evaluate the in vitro activity of tigecycline in comparison to tetracycline and other antimicrobial agents against clinical bacterial isolates recently collected from Latin American medical centers.

\section{Material and Methods}

\section{$\underline{\text { Organisms }}$}

A total of 1,326 clinical bacterial isolates collected from the Latin American region in 2000-2002 period were evaluated. The distribution of species was as following as: Staphylococcus aureus (505 strains), Streptococcus pneumoniae (269 strains), coagulase negative staphylococci (227 strains), Haemophilus influenzae (129 strains), Enterococcus spp. (80 strains), $\beta$-haemolytic streptococci (28 strains), viridans group streptococci (26 strains), Moraxella catarrhalis (54 strains), and Neisseria meningitides (8 strains). Only a single isolate per patient was evaluated. The isolates were identified to the species level by the participating medical center, and sent to the coordinating laboratory for identification confirmation and reference susceptibility testing.

\section{$\underline{\text { Medical centers }}$}

Clinical isolates of facultatively aerobic bacteria were collected in 11 Latin American laboratories distributed throughout 10 cities (six countries): São Paulo, Florianópolis, Porto Alegre and Brasília (only 2002), Brazil; Buenos Aires and San Isidro, Argentina; Santiago (two centers), Chile; Medellin, Colombia (only 2000); Caracas, Venezuela; and Mexico City, Mexico. The selection of participating centers was based on the principle that they should be sentinels in their respective geographic region. The participating medical centers were directed by a protocol to collect isolates from consecutive patients from specific sites of infections, including bloodstream infections, community-acquired respiratory infections, pneumonia in hospitalized patients, and skin and soft tissue infections.

\section{Susceptibility testing}

Antimicrobial susceptibility testing was performed using broth microdilution methods, as described by the Clinical Laboratory Standard Institute(CLSI, formerly NCCLS) [8]. Antimicrobial agents were obtained from their respective manufacturers as laboratory grade powder. Minimal inhibitory concentrations (MICs) results were interpreted according to NCCLS breakpoints [9]. A tigecycline susceptible breakpoint of $\leq 4 \mu \mathrm{g} / \mathrm{mL}$ for staphylococci and enterococci, and $\leq$ $2 \mu \mathrm{g} / \mathrm{mL}$ for other pathogens were used for comparative purposes only. Quality control measures were utilized by testing $S$. pneumoniae ATCC 49619, S. aureus ATCC 29213, Enterococcus faecalis ATCC 29212, Escherichia coli ATCC 25922, and Pseudomonas aeruginosa ATCC 27853.

\section{Results}

The largest number of isolates was collected from the Brazilian medical centers (573 isolates, 43.2\%), followed by the Chilean (377 isolates, 28.4\%) and Argentinean (264 isolates, 19.9\%) medical centers. These three countries contributed with $>90 \%$ of isolates (Table 1). Species tested in rank order of frequency were as follows: S. aureus (42.9\% oxacillin-resistant); S. pneumoniae (27.1\% penicillin-non-susceptible), CoNS (79.3\% oxacillin-resistant), $H$. influenzae (17.8\% $\beta$-lactamase-producers), Enterococcus spp. (10.3\% vancomycin-resistant), M. catarrhalis (92.6\% $\beta$-lactamase-producers), $\beta$-haemolytic streptococci (34.6\% erythromycin-non-susceptible), viridans group streptococci (30.8\% penicillin-non-susceptible), and Neisseria meningitides. 
Table 1. Frequency of pathogens tested for tigecycline susceptibility according to the country of isolation(SENTRY Antimicrobial Surveillance Program, Latin America, 2000-2002)

\section{Country}

\begin{tabular}{lccccccc} 
Organism & Argentina & Brazil & Chile & Colombia & Mexico & Venezuela & Total (\%) \\
\hline $\begin{array}{l}\text { Staphylococcus aureus } \\
\quad\end{array} \quad$ Oxacillin-susceptible & 53 & 144 & 63 & 7 & 5 & 16 & $288(21.7)$ \\
$\quad$ Oxacillin-resistant & 37 & 108 & 68 & 1 & 1 & 2 & $217(16.3)$ \\
& & & & & & & \\
CoNS & & & & & & & \\
$\quad$ Oxacillin-susceptible & 10 & 24 & - & 1 & 2 & 10 & $47(3.5)$ \\
$\quad$ Oxacillin-resistant & 33 & 108 & 8 & 4 & 16 & 11 & $180(13.5)$ \\
& & & & & & & \\
Enterococcus spp. & 15 & 41 & 10 & 1 & 1 & 12 & $80(6.0)$ \\
Streptococcus pneumoniae & 64 & 84 & 113 & - & - & 8 & $269(20.2)$ \\
b-haemolytic streptococci & 5 & 5 & 11 & 1 & 0 & 6 & $28(2.1)$ \\
Viridans group streptococci & 3 & 8 & 10 & - & 2 & 2 & $26(1.8)$ \\
$\begin{array}{l}\text { Haemophilus influenzae } \\
\text { Moraxella catarrhalis }\end{array}$ & 34 & 41 & 54 & - & - & - & $129(9.7)$ \\
Neisseria meningitidis & 9 & 10 & 33 & - & - & 2 & $54(4.0)$ \\
\end{tabular}

Total (\%)

$264(19.9) 573(43.2) 377(28.4) \quad 15(1.1) 26(1.9) \quad 69(5.2) \quad 1,326(100.0)$

${ }^{a}$ CoNS: Coagulase-negative staphylococci.

Theantimicrobial activity of tigecycline was compared to selected antimicrobial agents (Table 2). Tigecycline was highly active against both oxacillin-resistant andsusceptible $S$. aureus $\left(\mathrm{MIC}_{50}, 0.25 \mu \mathrm{g} / \mathrm{mL}\right.$ and $\mathrm{MIC}_{90}$, $0.5 \mu \mathrm{g} / \mathrm{mL}$ for both groups). More than $99 \%$ of the strains were inhibited at $\leq 0.5 \mu \mathrm{g} / \mathrm{mL}$ of tigecycline. Similarly to S. aureus, both oxacillin-resistant and -susceptible coagulase-negative staphylococci were very susceptible to tigeclycline $\left(\mathrm{MIC}_{50}, 0.25 \mu \mathrm{g} / \mathrm{mL}\right.$ and $\mathrm{MIC}_{90}, 0.5 \mu \mathrm{g} /$ $\mathrm{mL}$ for both). Vancomycin and linezolid were the only antimicrobial agents active against all staphylococcal strains at the susceptible breakpoint. Tigecycline $\left(\mathrm{MIC}_{50}\right.$, $0.25 \mu \mathrm{g} / \mathrm{mL}$ ) was at least four-fold more potent than vancomycin $\left(\mathrm{MIC}_{50}, 1 \mu \mathrm{g} / \mathrm{mL}\right.$ ) or linezolid $\left(\mathrm{MIC}_{50}, 1-2\right.$ $\mu \mathrm{g} / \mathrm{mL}$ ) against oxacillin-resistant staphylococci. Tigecycline was also highly active against tetracycline- resistant staphylococci. Co-resistance among tetracycline, erythromycin, and fluoroquinolones was noticed within the subsets of oxacillin-resistant staphylococci and did not affect tigecycline in vitro activity against staphylococci.

Although tigecycline and linezolid inhibited 100\% of the Enterococcus spp. strains tested, tigecycline $\left(\mathrm{MIC}_{50}, 0.25 \mu \mathrm{g} / \mathrm{mL}\right.$ ) was eight-fold more potent than linezolid ( $\mathrm{MIC}_{50}, 2 \mu \mathrm{g} / \mathrm{mL}$ ). The vast majority of Enterococcus spp. were susceptible to ampicillin $\left(\mathrm{MIC}_{50}, \leq 1 \mu \mathrm{g} / \mathrm{mL}\right.$; 88.8\% susceptible) and resistant to quinupristin/dalfopristin ( $\mathrm{MIC}_{50}, 8 \mu \mathrm{g} / \mathrm{mL} ; 13.8 \%$ susceptible), reflecting the preponderance of $E$. faecalis in the collection. Tigecycline showed excellent potency against both vancomycin- and tetracyclineresistant Enterococcus spp. 
Table 2. Antimicrobial activity of tigecycline in comparison to selected antimicrobial agents against 1,326 pathogen isolates from Latin American medical centers (SENTRY Antimicrobial Surveillance Program, 2000-2002)

\begin{tabular}{|c|c|c|c|}
\hline \multirow{2}{*}{$\begin{array}{l}\text { Organism/antimicrobial agent } \\
\text { (no. tested) }\end{array}$} & \multicolumn{2}{|c|}{$\operatorname{MIC}(\mu \mathrm{g} / \mathrm{mL})^{\mathrm{a}}$} & \multirow[b]{2}{*}{$\%$ susceptible } \\
\hline & $\mathrm{MIC}_{50}$ & $\mathrm{MIC}_{90}$ & \\
\hline \multicolumn{4}{|l|}{$\overline{\text { Staphylococcus aureus }}$} \\
\hline \multicolumn{4}{|l|}{ Oxacillin-susceptible (288) } \\
\hline Tigecycline & 0.25 & 0.5 & $100.0^{c}$ \\
\hline Tetracycline & $\leq 4$ & $>8$ & 90.2 \\
\hline Erythromycin & 0.25 & $>8$ & 85.1 \\
\hline Ciprofloxacin & 0.25 & 0.5 & 96.9 \\
\hline Quinupristin/Dalfopristin & 0.25 & 0.5 & 100.0 \\
\hline Teicoplanin & 0.5 & 1 & 99.7 \\
\hline Vancomycin & 1 & 1 & 100.0 \\
\hline Linezolid & 2 & 2 & 100.0 \\
\hline \multicolumn{4}{|l|}{ Oxacillin-resistant (217) } \\
\hline Tigecycline & 0.25 & 0.5 & $100.0^{c}$ \\
\hline Tetracycline & 8 & $>8$ & 49.8 \\
\hline Erythromycin & $>8$ & $>8$ & 7.4 \\
\hline Ciprofloxacin & $>2$ & $>2$ & 4.6 \\
\hline Quinupristin/Dalfopristin & 0.5 & 1 & 100.0 \\
\hline Teicoplanin & 1 & 2 & 99.1 \\
\hline Vancomycin & 1 & 1 & 100.0 \\
\hline Linezolid & 2 & 2 & 100.0 \\
\hline \multicolumn{4}{|l|}{ CoNS } \\
\hline \multicolumn{4}{|l|}{ Oxacillin-susceptible (47) } \\
\hline Tigecycline & 0.25 & 0.5 & $100.0^{c}$ \\
\hline Tetracycline & 4 & $>8$ & 76.6 \\
\hline Erythromycin & 0.25 & $>8$ & 74.4 \\
\hline Ciprofloxacin & 0.25 & 0.25 & 95.3 \\
\hline Quinupristin/Dalfopristin & 0.25 & 0.25 & 100.0 \\
\hline Teicoplanin & 1 & 2 & 100.0 \\
\hline Vancomycin & 1 & 2 & 100.0 \\
\hline Linezolid & 1 & 1 & 100.0 \\
\hline \multicolumn{4}{|l|}{ Oxacillin-resistant (180) } \\
\hline Tigecycline & 0.25 & 0.5 & $100.0^{c}$ \\
\hline
\end{tabular}




\begin{tabular}{|c|c|c|c|}
\hline Tetracycline & $\leq 4$ & $>8$ & 78.3 \\
\hline Erythromycin & $>8$ & $>8$ & 25.0 \\
\hline Ciprofloxacin & 2 & $>2$ & 47.8 \\
\hline Quinupristin/Dalfopristin & 0.25 & 0.5 & 96.7 \\
\hline Teicoplanin & 2 & 8 & 93.3 \\
\hline Vancomycin & 1 & 2 & 100.0 \\
\hline Linezolid & 1 & 2 & 100.0 \\
\hline \multicolumn{4}{|c|}{ Streptococcus pneumoniae (269) } \\
\hline Tigecycline & 0.12 & 0.12 & $100.0^{\mathrm{c}}$ \\
\hline Tetracycline & $\leq 4$ & $>8$ & $83.6^{f}$ \\
\hline Erythromycin & 0.25 & 1 & 87.7 \\
\hline Penicillin & 0.03 & 2 & 72.9 \\
\hline Ceftriaxone & 0.25 & 1 & $98.5^{\mathrm{e}}$ \\
\hline Gatifloxacin & 0.25 & 0.25 & 100.0 \\
\hline Quinupristin/Dalfopristin & 0.25 & 0.5 & 100.0 \\
\hline Teicoplanin & 0.12 & 0.12 & 100.0 \\
\hline Vancomycin & 0.25 & 0.5 & 100.0 \\
\hline Linezolid & 1 & 1 & 100.0 \\
\hline \multicolumn{4}{|l|}{$\beta$-haemolytic streptococci (28) } \\
\hline Tigecycline & 0.12 & 0.12 & $100.0^{c}$ \\
\hline Tetracycline & $>8$ & $>8$ & 46.4 \\
\hline Erythromycin & 0.06 & 1 & 89.3 \\
\hline Penicillin & 0.03 & 0.06 & 96.4 \\
\hline Gatifloxacin & 0.25 & 0.5 & 96.4 \\
\hline Quinupristin/Dalfopristin & 0.25 & 0.5 & 100.0 \\
\hline Teicoplanin & 0.12 & 0.12 & 100.0 \\
\hline Vancomycin & 0.25 & 0.5 & 100.0 \\
\hline Linezolid & 1 & 1 & 100.0 \\
\hline \multicolumn{4}{|c|}{ Viridans group streptococci (26) } \\
\hline Tigecycline & 0.12 & 0.25 & $100.0^{c}$ \\
\hline Tetracycline & $\leq 4$ & $>8$ & 73.1 \\
\hline Erythromycin & 0.06 & 2 & 65.4 \\
\hline Penicillin & 0.12 & 2 & 69.2 \\
\hline Gatifloxacin & 0.25 & 0.5 & 96.2 \\
\hline Quinupristin/Dalfopristin & 0.5 & 1 & 92.3 \\
\hline Teicoplanin & 0.12 & 0.12 & 100.0 \\
\hline Vancomycin & 0.5 & 1 & 100.0 \\
\hline Linezolid & 1 & 1 & 100.0 \\
\hline \multicolumn{4}{|l|}{ Enterococcus spp. (80) } \\
\hline Tigecycline & 0.25 & 0.5 & $100.0^{c}$ \\
\hline Tetracycline & $>8$ & $>8$ & 27.5 \\
\hline Ampicillin & $\leq 1$ & 16 & 88.8 \\
\hline
\end{tabular}




\begin{tabular}{lccr}
\hline Ciprofloxacin & 1 & $>2$ & 55.0 \\
Quinupristin/Dalfopristin & 8 & 8 & 13.8 \\
Teicoplanin & 0.25 & 1 & 92.5 \\
Vancomycin & 2 & 8 & 89.7 \\
Linezolid & 2 & 2 & 100.0 \\
& & & \\
Haemophilus influenzae (129) & & & \\
Tigecycline & 0.25 & 0.25 & $100.0^{\mathrm{c}}$ \\
Tetracycline & $\leq 4$ & $\leq 4$ & $96.1^{\mathrm{f}}$ \\
Azithromycin & 0.5 & 1 & 100.0 \\
Clarithromycin & 8 & 8 & 93.0 \\
Ampicillin & 0.5 & 1 & 82.2 \\
Amoxicillin/Clavulanate & 0.5 & 1 & 100.0 \\
Ceftriaxone & 0.008 & 0.016 & 100.0 \\
Ciprofloxacin & $\leq 0.03$ & $\leq 0.03$ & 100.0 \\
Chloramphenicol & $\leq 2$ & $\leq 2$ & 94.6 \\
& & & \\
Moraxella catarrhalis (54) & & & \\
Tigecycline & & 0.5 & $100.0^{c}$ \\
Tetracycline & 0.12 & $\leq 4$ & 100.0 \\
Azithromycin & $\leq 4$ & $\leq 0.12$ & 100.0 \\
Clarithromycin & $\leq 0.12$ & $\leq 0.25$ & 100.0 \\
Ampicillin & $\leq 0.25$ & 2 & 7.4 \\
Amoxicillin/Clavulanate & $\leq 0.5$ & 0.25 & 100.0 \\
Ceftriaxone & 0.12 & 0.5 & 100.0 \\
Ciprofloxacin & 0.12 & $\leq 2$ & 100.0 \\
Chloramphenicol & $\leq 0.03$ & 100.0 \\
\hline
\end{tabular}

${ }^{\mathrm{a}}$ Minimal inhibitory concentration determined by the broth microdilution technique [8]. ${ }^{\text {PPercentage of }}$ susceptibility calculated using the NCCLS breakpoints [9]. ${ }^{\mathrm{C}}$ A susceptible breakpoint of $\leq 4 \mu \mathrm{g} / \mathrm{mL}$ was used

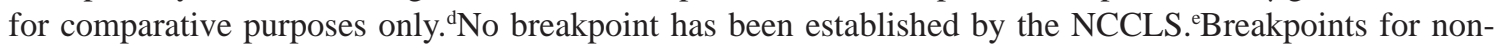
meningitis were applied. Includes susceptible and intermediate. ${ }^{\text {g}}$ Breakpoints for $H$. influenzae were used.

Against S. pneumoniae, tigecycline, gatifloxacin, quinupristin/dalfopristin, vancomycin, teicoplanin, and linezolid showed a susceptibility rate of $100.0 \%$. Four S. pneumoniae isolates were not susceptible to ceftriaxone when non-meningitis breakpoints were applied. Tigecycline ( $\mathrm{MIC}_{50}$, $0.12 \mu \mathrm{g} / \mathrm{mL}$ ) was as potent as teicoplanin $\left(\mathrm{MIC}_{50}\right.$, $0.12 \mu \mathrm{g} / \mathrm{mL}$ ) and two-fold more potent than ceftriaxone ( $\mathrm{MIC}_{50}, 0.25 \mu \mathrm{g} / \mathrm{mL}$ ), gatifloxacin $\left(\mathrm{MIC}_{50}, 0.25 \mu \mathrm{g} / \mathrm{mL}\right.$ ), and vancomycin (MIC $_{50}$, $0.25 \mu \mathrm{g} / \mathrm{mL}$ ) against $S$. pneumoniae. Tigecycline was also highly active against $S$. pneumoniae, including isolates resistant to penicillin and/or tetracycline and/or erythromycin.

Tigecycline demonstrated excellent activity against viridans group streptococci $\left(\mathrm{MIC}_{50 / 90}, \leq 0.12 / 0.25 \mu \mathrm{g} /\right.$ $\mathrm{mL}$ ) and $\beta$-haemolytic streptococci $\left(\mathrm{MIC}_{50 / 90}, \leq 0.12\right.$ $\mu \mathrm{g} / \mathrm{mL}$ ), whereas the corresponding $\mathrm{MIC}_{90}$ values for tetracycline were $>8 \mu \mathrm{g} / \mathrm{mL}$. Tigecycline, vancomycin, teicoplanin, and linezolid (100.0\% susceptible) exhibited excellent coverage against these pathogens. No cross-resistance between tigecycline and any of the antimicrobial agents tested was observed among streptococcal isolates. 
Table 3. Tigecycline MIC distribution among 1,326 pathogens from the SENTRY Antimicrobial Surveillance Program (Latin America, 2000-2002)

\begin{tabular}{|c|c|c|c|c|c|}
\hline \multirow[t]{2}{*}{ Organism (n tested) } & \multicolumn{5}{|c|}{ No. of isolates (cumulative \%) inhibited at MIC $(\mu \mathrm{g} / \mathrm{mL})$} \\
\hline & $\leq \mathbf{0 . 1 2}$ & 0.25 & 0.5 & 1 & 2 \\
\hline \multicolumn{6}{|l|}{ Staphylococcus aureus } \\
\hline Oxacillin-susceptible (288) & $111(38.5)$ & $94(71.2)$ & $81(99.3)$ & $2(100.0)$ & - \\
\hline Oxacillin-resistant (217) & $63(29.1)$ & $100(75.1)$ & $51(98.6)$ & $2(99.5)$ & $1(100.0)$ \\
\hline \multicolumn{6}{|l|}{ CoNS $^{\mathrm{a}}$} \\
\hline Oxacillin-susceptible (47) & 15 (31.9) & $15(63.8)$ & $14(93.6)$ & $3(100.0)$ & - \\
\hline Oxacillin-resistant (180) & $41(22.8)$ & $55(53.3)$ & $70(92.2)$ & $13(99.4)$ & $1(100.0)$ \\
\hline Streptococcus pneumoniae (269) & $268(99.6)$ & $1(100.0)$ & - & - & - \\
\hline Haemophilus influenzae (129) & $8(6.2)$ & $28(27.9)$ & 72 (83.7) & 17 (96.9) & $4(100.0)$ \\
\hline Enterococcus spp. (80) & $37(43.3)$ & $23(75.0)$ & $20(100.0)$ & - & - \\
\hline Moraxella catarrhalis (54) & $31(57.4)$ & 17 (88.9) & $6(100.0)$ & - & - \\
\hline$\beta$-haemolytic streptococci (28) & $28(100.0)$ & - & - & - & - \\
\hline Viridans group streptococci (26) & $23(88.5)$ & $1(92.3)$ & $2(100.0)$ & - & - \\
\hline Neisseria meningitidis (8) & $8(100)$ & - & - & - & - \\
\hline
\end{tabular}

a.CoNS: Coagulase-negative staphylococci.

Nearly $18.0 \%$ and $93.0 \%$ of the $H$. influenzae and $M$. catarrhalis strains produced $\beta$-lactamase, respectively. Haemophilus influenzae $\left(\mathrm{MIC}_{50 / 90}\right.$, $0.25 \mu \mathrm{g} / \mathrm{mL})$ and $M$. catarrhalis $\left(\mathrm{MIC}_{50 / 90}, \leq 0.12 /\right.$ $0.5 \mu \mathrm{g} / \mathrm{mL}$ ) isolates were very susceptible to tigecycline (100.0\% susceptible), including $\beta$ lactamase producing isolates. Only eight $N$. meningitidis isolates were tested. Seven of them were collected from a Chilean medical center. All of the $N$. meningitidis showed tigecycline MIC at concentrations $\leq 0.12 \mu \mathrm{g} / \mathrm{mL}$. The tigecycline MIC distribution among 1,326 pathogens tested is shown in Table 3. The tigecycline MICs distributions were not influenced by co-phenotypes of resistance, such as oxacillin-resistance among staphylococci, penicillin-resistance among $S$. pneumoniae or glycopeptide resistance among Enterococcus spp. The vast majority of isolates tested (1320 of 1326 or $99.5 \%$ ) were inhibited at a concentration of $\leq 1$ $\mu \mathrm{g} / \mathrm{mL}$ of tigecycline.

\section{Discussion}

Tigecycline, a novel glycylcycline antibiotic, exhibits strong activity against Gram-positive, Gram-negative, aerobic, anaerobic, and atypical bacterial species, including many resistant pathogens, i.e., vancomycinresistant enterococci, methicillin-resistant $S$. aureus and penicillin-resistant $S$. pneumoniae [10-13]. In addition, tigecycline has shown excellent activity against most Gram-negative pathogens, including Enterobacteriaceae, Acinetobacter spp., Stenotrophomonas maltophilia [10,11], Haemophilus influenzae, and Neisseria spp. [14]. Tigecycline's expanded broad-spectrum activity is further evidenced by its activity against Legionella pneumophila [15], Chlamydia [16], rapidly growing nontuberculosis mycobacteria [17] and anaerobes [18]. On the other hand, tigecycline has demonstrated limited activity against $P$. aeruginosa and Proteae isolates $[5,10,11]$. 
The results of two randomized trials assessing the clinical efficacy of tigecycline were recently released [19]. The studies evaluated tigecycline versus vancomycin plus aztreonam to treat complicated skin and skin structure infections (cSSSI), and tigecycline versus imipenem plus cilastatin to treat complicated intrabdominal infections (cIAI). In the first trial, 274 patients received tigecycline and 269 received vancomycin plus aztreonam. The clinical cure rate of cSSSI between the two groups was not statistically different ( $84.3 \%$ versus $86.9 \%$, respectively). The cIAI trial comprised 404 and 413 patients treated with tigecycline and imipenem plus cilastatin, respectively. Clinical cure was again not statistically distinct (86.6\% of the tigecycline group and in $84.4 \%$ of those on combination therapy). In both trials, the most frequently reported adverse events for patients on tigecycline were nausea and vomiting [19].

Our results agree with previous in vitro studies [5,10-14] and emphasize that tigecycline has excellent activity and spectrum against Gram-positive bacteria, including multi-drug resistant strains, and bacterial pathogens causing community-acquired respiratory tract infections isolated from patients hospitalized in Latin American medical centers. These results, associated with the tigecycline clinical efficacy data already published, suggest that tigecycline may have an important role in the treatment of severely ill patients, especially in an environment with high antimicrobial resistance levels such as the Latin American region.

\section{References}

1. Chopra I., Hawkey P.M., Hinton M. Tetracyclines, molecular and clinical aspects. J Antimicrob Chemother 1992;29:245-77.

2. Speer B.S., Shoemaker N.B., Salyers A.A. Bacterial resistance to tetracycline: mechanisms, transfer, and clinical significance. Clin Microbiol Rev 1992;5:387-99.

3. McMurry L.M., Park B.H., Burdett V., Levy S.B. Energydependent efflux mediated by class L (tetL) tetracycline resistance determinant from streptococci. Antimicrob Agents Chemother 1987;31:1648-50.

4. Levy S.B. Evolution and spread of tetracycline resistance determinants. J Antimicrob Chemother 1989;24:1-3.
5. Petersen P.J., Jacobus N.V., Weiss W.J., et al. In vitro and in vivo antibacterial activities of a novel glycylcycline, the 9-t-butylglycylamido derivative of minocycline (GAR936). Antimicrob Agents Chemother 1999;43:738-44.

6. Sum P.E., Petersen P. Synthesis and structure-activity relationship of novel glycylcycline derivatives leading to the discovery of GAR-936. Bioorg Med Chem Lett 1999;9:1459-62.

7. Bergeron J., Ammirati M., Danley D., et al. Glycylcyclines bind to the high-affinity tetracycline ribosomal binding site and evade Tet(M)- and Tet(O)-mediated ribosomal protection. Antimicrob Agents Chemother 1996;40:2226-8.

8. National Committee for Clinical Laboratory Standards. Methods for dilution antimicrobial susceptibility tests for bacteria that grow aerobically; approved standard Sixth edition. Approved document M7-A6. Wayne, PA:NCCLS. 2003.

9. National Committee for Clinical Laboratory Standards. Performance standards for antimicrobial susceptibility testing, $15^{\text {th }}$ informational supplement M100-S15. Wayne, PA:NCCLS. 2005.

10. Gales A.C., Jones R.N. Antimicrobial activity and spectrum of the new glycylcycline, GAR-936 tested against 1,203 recent clinical bacterial isolates. Diagn Microbiol Infect Dis 2000;36:19-36.

11. Milatovic D., Schmitz F.J., Verhoef J., Fluit A.C. Activities of the glycylcycline tigecycline (GAR-936) against 1,924 recent European clinical bacterial isolates. Antimicrob Agents Chemother 2003;47:400-4.

12. Fritsche T.R., Jones R.N. Antimicrobial activity of tigecycline (GAR-936) tested against 3498 recent isolates of Staphylococcus aureus recovered from nosocomial and community-acquired infections. Int J Antimicrob Agents 2004;24:567-71.

13. Fritsche T.R., Kirby J.T., Jones R.N. In vitro activity of tigecycline (GAR-936) tested against 11,859 recent clinical isolates associated with community-acquired respiratory tract and Gram-positive cutaneous infections. Diagn Microbiol Infect Dis 2004;49:201-9.

14. Deshpande L.M., Gales A.C., Jones R.N. GAR-936 (9-tbutylglycylamido-minocycline) susceptibility test development for streptococci, Haemophilus influenzae and Neisseria gonorrhoeae: preliminary guidelines and interpretive criteria. Int J Antimicrob Agents 2001;18:29-35.

15. Edelstein P.H., Weiss W.J., Edelstein M.A. Activities of tigecycline (GAR-936) against Legionella pneumophila in vitro and in guinea pigs with $L$. pneumophila pneumonia. Antimicrob. Agents Chemother 2003;47:533-40.

16. Roblin P.M., Hammerschlag M.R. In vitro activity of GAR936 against Chlamydia pneumoniae and Chlamydia trachomatis. Int J Antimicrob Agents 2000;16:61-3. 
17. Wallace R.J., Brown-Elliott Jr.B.A., Crist C.J., et al. Comparison of the in vitro activity of the glycylcycline tigecycline (formerly GAR-936) with those of tetracycline, minocycline, and doxycycline against isolates of nontuberculous mycobacteria. Antimicrob. Agents Chemother 2002;46:3164-7.

18. Petersen P.J., Weiss W.J., Labthavikul P.. The postantibiotic effect and time-kill kinetics of the glycylcyclines, GAR-936 (TBG-MINO) and (PAMMINO). Program Abstr. 38th Intersci. Conf. Antimicrob. Agents Chemother 1998;abstr. F-132.

19. McConnell J. Results of tigecycline trials announced. Lancet Infect Dis 2004;4:717. 